\title{
Unplanned 30-Day Readmissions in Orthopaedic Trauma
}

Introduction: 30-day readmission is increasingly used as a hospital quality metric. The objective of this study was to describe the patient factors associated with unplanned 30-day hospital readmission of orthopaedic trauma patients.

Methods: A statewide observational study was undertaken using data from all acute hospitals in California. All hospital inpatients with a primary diagnosis of fracture or dislocation (ICD-9-CM 800-829) were included, except for those with isolated injuries to the skull, face, or ribs. The primary outcome measure was unplanned 30day readmission to any hospital in California.

Results: 416,568 trauma admissions were available for analysis. The overall readmission rate was $6.5 \%$, and $27.3 \%$ of readmitted patients presented to a different hospital. Factors significantly associated with readmission were male sex (OR 1.23, 95\% CI 1.19-1.27), age 46-65 (2.61 [2.27-2.99]), black race (1.19 [1.111.27]), entitlement to publicly funded healthcare (1.38 [1.25-1.52]), Charlson Comorbidity Index $\geq 2$ (1.84 [1.79-1.90]), discharge against medical advice (3.13 [2.67-3.68]), and spinal fracture (1.42 [1.34-1.49]). Major reasons for readmission included: cardiopulmonary disease (25.6\%), infections (20.1\%), musculoskeletal problems (18.1\%), and procedural complications (12.0\%).

Conclusions: Many orthopaedic trauma readmissions are potentially unrelated to the initial hospitalization. Penalties for unplanned readmissions risk penalizing hospitals that serve disadvantaged communities and treat a high proportion of trauma patients. 


\section{Introduction}

Unplanned readmission to hospital is increasingly used as a health outcome measure $^{1,2}$. Up to $20 \%$ of Medicare beneficiaries are re-hospitalized within 30 days of discharge from an acute care hospital, at an annual cost of $\$ 17$ billion ${ }^{3,4}$. There is evidence that many readmissions are preventable, particularly given considerable variation between healthcare providers and evidence from randomized controlled trials that specific interventions reduce readmission rates 5,6 . Reducing unplanned readmissions presents an opportunity both to improve healthcare quality and reduce $\operatorname{cost}^{2}$. For this reason, some healthcare systems use readmission rates as a quality metric for the purposes of calculating healthcare payments ${ }^{7,8}$. In the United States, the Hospital Readmissions Reduction Program (HRRP) penalizes hospitals up $3 \%$ of total Medicare payments for excess risk-adjusted readmissions across a range of medical conditions, including acute myocardial infarction, congestive cardiac failure, and pneumonia 8 .

Although there are plans to extend the HRRP to include specific surgical populations ${ }^{1}$, it remains unclear whether readmission rates measure quality or simply reflect socioeconomic factors beyond the influence of individual hospitals. Factors associated with readmission include living alone, limited education, poor family support, and ethnic minority status. ${ }^{9}$ Perhaps unsurprisingly, the first wave of HRRP penalties overwhelmingly penalized "safety net" hospitals, i.e. those treating high numbers of disadvantaged and ethnic minority patients ${ }^{8}$. 
There is increasing interest in using 30 -day readmission rates as a quality metric in trauma care. This is important as trauma predominantly affects patients with independent risk factors (such as low socioeconomic status) for unplanned readmission. However, a recent systematic review ${ }^{10}$ identified only one singlecenter study that explored predictors for readmission of orthopaedic trauma patients ${ }^{11}$.

Our study used a comprehensive statewide population database to identify risk factors for unplanned 30-day readmission in the orthopaedic trauma setting. This database permitted longitudinal follow-up of patients, including those that were readmitted to a hospital other than the institution at which they were initially treated.

\section{Methods}

A statewide administrative database was analyzed to identify risk factors and reasons for unplanned hospital readmission within 30 days of discharge following traumatic injury. The protocol was approved by our hospital institutional review board.

\section{Data source}

Data were extracted from the California State Inpatient Database (SID) 2007-2011. This resource is part of the family of databases developed for the Healthcare Cost and Utilization Project (HCUP), which aims to create national resource of patient- 
and provider-level data for administrative and research purposes. The SID contains 98\% of inpatient discharge records from hospitals in California, regardless of payment source ${ }^{12}$. It includes data on over a hundred demographic and clinical variables for over four million inpatient episodes per year. Unique identifiers within the SID allow individuals to be tracked between admissions, even when these are to different hospitals. Outpatient and emergency department visits that do not lead to readmission are not recorded within the SID.

The California SID was linked to the American Hospital Association Annual Survey Database (2012), which provides additional details necessary for analysis of hospital-level factors.

\section{Selection of cases}

All orthopaedic trauma cases were identified using International Coding of Diseases, $9^{\text {th }}$ Revision (ICD-9-CM) codes. These included all fractures (800-829), except for those related to the skull (800-801), face (802-804) and ribs (807); and all dislocations (830-839) except for those of the jaw (830). No other limits were applied in terms of specific patient demographics (e.g. age) or injury type.

\section{Categories and definitions}

Hospitals were designated as teaching hospitals if approved for residency training by the Accreditation Council for Graduate Medical Education (ACGME). 
The Charlson Comorbidity Indices (CCI) and Injury Severity Scores (ISS) were determined from ICD-9 codes using the CHARLSON and ICDPIC modules respectively within Stata. Patient groups were categorized by age $(0-18,19-45,46-$ 65 , $\geq 65)$, Charlson score $(<2, \geq 2)$, injury severity score $(<15, \geq 15)$, and injury type. Injuries were classified by ICD-9 codes as upper limb (810-819), spine (805-806), pelvis (808), hip (820), femur (821), tibia (823), foot and ankle (824-827), other fracture $(809,822,828,829)$, and dislocation (831-839).

\section{Statistical analysis}

Differences between groups were tested using Chi square tests for categorical variables and Wilcoxon rank-sum tests for continuous variables. Multivariable logistic regression models were used to identify factors independently associated with 30-day readmission. All analyses were performed using Stata v.13 (StataCorp, College Station, TX). The threshold for statistical significance was set at $\mathrm{P}<0.05$.

\section{Results}

There were 416,568 orthopaedic trauma admissions to 391 different hospitals in California between 2007 and 2011, of which 27,008 (6.5\%) were readmitted within 30 days of discharge.

Table 1 shows that the study population was predominantly older (age $\geq 65$ ), male, white, and funded by public insurance. Tables 1 and 2 present the characteristics that were associated with 30-day readmission. In the multivariable regression 
model, the strongest independent predictors of readmission included male sex (OR 1.23, 95\% CI 1.19-1.27), age 46-65 (2.61, 2.27-2.99), black race (1.19, 1.11-1.27), entitlement to publicly funded healthcare $(1.38,1.25-1.52)$, CCI $\geq 2(1.84,1.79-1.90)$, ISS $\geq 15(1.10,1.01-1.19)$, discharge against medical advice $(3.13,2.67-3.68)$ and spinal fracture $(1.42,1.34-1.49)$.

Table 3 categorizes the reasons for readmission. The most frequent reasons were cardiopulmonary (25.6\%), followed by infection (20.1\%), musculoskeletal problems (18.1\%), and procedural complications (12.0\%). Importantly, 8,576 patients $(27.3 \%$ of readmissions) presented to a different hospital to the institution at which they were initially treated (Table 4). Although those presenting to a different hospital had a longer hospital stay (5.7 vs 6.4 days, $\mathrm{p}=0.002$ ), there were no differences in mortality (6.0 vs $5.9, \mathrm{p}=0.776$ ), complication rate ( 22.1 vs $21.4 \%, \mathrm{p}=0.182$ ), or subsequent 30 -day readmission $(17.9 \%$ vs $18.3 \%$, $\mathrm{p}=0.408)$ between these groups.

\section{Discussion}

The only previous study to explore factors associated with 30-day readmission within the orthopaedic trauma setting was based on the experience of a single center ${ }^{11}$. Our study is the first comprehensive statewide description of readmissions across the orthopaedic trauma population. This is an important step towards informing future discussions about using these as a quality metric in the trauma setting. 
There was an overall readmission rate of $6.5 \%$, which is comparable to that reported in other surgical populations ${ }^{13}$. However, it is higher than the $5.4 \%$ previously reported in orthopaedic trauma ${ }^{11}$, possibly because our study also captured readmissions to other institutions. We found that many readmitted patients $(27.3 \%)$ presented to other hospitals, which is consistent with evidence from Canada ${ }^{13}$. This finding quantifies the number of patients that are missed by single center studies of 30-day readmissions in other orthopaedic populations ${ }^{10}$.

Significant predictors of readmission were similar to those found by other studies: publicly funded healthcare, comorbidities, and discharge disposition ${ }^{14-18}$. Black race was also associated with readmission. Although racial disparities in trauma have been documented previously ${ }^{19}$ they are less striking than in other surgical populations $\mathbf{s}^{20,21}$.

The most common reasons for readmission were related to cardiopulmonary (e.g. heart failure, chronic obstructive pulmonary disease) and infectious (e.g. cellulitis, pneumonia, urinary tract infection) diagnoses. Procedural complications accounted for only $12.0 \%$ of readmissions. These observations suggest that improving the long-term medical management of orthopaedic trauma patients could be an important strategy for reducing readmissions.

The limitations of our study are those of all retrospective analyses of administrative datasets. Although SID data goes through a series of internal validation steps ${ }^{12}$, 
individual patients cannot be identified and so it was not possible for us to externally validate the classification and coding of cases within the database. The analysis was also confined by variables recorded in the dataset. For example, it is possible that other factors (e.g. education status) might independently predict readmission risk but these could not be directly tested in our study. However, the SID did provide a unique opportunity to perform a population-based analysis tracking all inpatient admissions within a whole state across multiple years.

\section{Conclusion}

It is possible that readmissions will ultimately be adopted as a quality metric in trauma care and efforts to reduce unplanned readmissions could improve care while reducing costs. Our analysis supports the concern that financial penalties could unfairly penalize hospitals serving disadvantaged populations at high risk of traumatic injury, and so potentially exacerbate poor outcomes. It also highlights the need to create tools that equitably adjust for patient characteristics to reduce the risk of penalizing hospitals that treat a high proportion of trauma patients. 


\section{References}

1. Girotti ME, Shih T, Dimick JB. Health policy update: rethinking hospital readmission as a surgical quality measure. JAMA Surg. 2014; 149(8):757-8.

2. Dimick JB, Ghaferi AA. Hospital readmission as a quality measure in surgery. JAMA. 2015; 313(5):512-3.

3. Jencks SF, Williams MV, Coleman EA. Rehospitalizations among patients in the Medicare fee-for-service program. $N$ Engl J Med. 2009; 360(14):1418-28.

4. Shams I, Ajorlou S, Yang K. A predictive analytics approach to reducing 30day avoidable readmissions among patients with heart failure, acute myocardial infarction, pneumonia, or COPD. Health Care Manag Sci. 2015; 18(1):19-34.

5. Krumholz HM, Amatruda J, Smith GL, et al. Randomized trial of an education and support intervention to prevent readmission of patients with heart failure. J Am Coll Cardiol. 2002; 39(1):83-9.

6. Coleman EA, Parry C, Chalmers S, et al. The care transitions intervention: results of a randomized controlled trial. Arch Intern Med. 2006; 166(17):1822-8.

7. Blunt I, Bardsley M, Grove A, et al. Classifying emergency 30-day readmissions in England using routine hospital data 2004-2010: what is the scope for reduction? Emerg Med J. 2015; 32(1):44-50.

8. Joynt KE, Jha AK. Characteristics of hospitals receiving penalties under the Hospital Readmissions Reduction Program. JAMA. 2013; 309(4):342-3. 
9. Arbaje AI, Wolff JL, $\mathrm{Yu}$ Q, et al. Postdischarge environmental and socioeconomic factors and the likelihood of early hospital readmission among community-dwelling Medicare beneficiaries. Gerontologist. 2008; 48(4):495-504.

10. Bernatz JT, Tueting JL, Anderson PA. Thirty-day readmission rates in orthopedics: a systematic review and meta-analysis. PLoS One. 2015; 10(4):e0123593.

11. Hageman MG, Bossen JK, Smith RM, et al. Predictors of readmission in orthopaedic trauma surgery. J Orthop Trauma. 2014; 28(10):e247-9.

12. Healthcare Cost and Utilization Project. Overview of the State Inpatient Databases (SID) 2015. Available at: http://www.hcupus.ahrq.gov/sidoverview.jsp. Accessed 27 November 2015.

13. Moore L, Stelfox HT, Turgeon AF, et al. Rates, patterns, and determinants of unplanned readmission after traumatic injury: a multicenter cohort study. Ann Surg. 2014; 259(2):374-80.

14. Fawcett VJ, Flynn-O'Brien KT, Shorter Z, et al. Risk factors for unplanned readmissions in older adult trauma patients in Washington State: a competing risk analysis. J Am Coll Surg. 2015; 220(3):330-8.

15. Vachon $\mathrm{CM}$, Aaland $\mathrm{M}$, Zhu TH. Readmission of trauma patients in a nonacademic Level II trauma center. J Trauma Acute Care Surg. 2012; 72(2):531-6. 
16. Marcin JP, Romano PS. Impact of between-hospital volume and withinhospital volume on mortality and readmission rates for trauma patients in California. Crit Care Med. 2004; 32(7):1477-83.

17. Laupland KB, Svenson LW, Grant V, et al. Long-term mortality outcome of victims of major trauma. Injury. 2010; 41(1):69-72.

18. Battistella FD, Torabian SZ, Siadatan KM. Hospital readmission after trauma: an analysis of outpatient complications. J Trauma. 1997; 42(6):1012-6.

19. Haider AH, Weygandt PL, Bentley JM, et al. Disparities in trauma care and outcomes in the United States: a systematic review and meta-analysis. $J$ Trauma Acute Care Surg. 2013; 74(5):1195-205.

20. Tepas JJ, 3rd, Pracht EE, Orban BL, et al. Insurance status, not race, is a determinant of outcomes from vehicular injury. J Am Coll Surg. 2011; 212(4):722-7.

21. Salim A, Ottochian M, DuBose J, et al. Does insurance status matter at a public, level I trauma center? J Trauma. 2010; 68(1):211-6. 